ACCEPTED B Y ApJ.

Preprint typeset using LTEX style emulateapj v. 08/22/09

\title{
SUPERMODEL ANALYSIS OF GALAXY CLUSTERS
}

\author{
R. Fusco-Femiano ${ }^{1}$, A. CAVAliere ${ }^{2}$, A. LAPI ${ }^{2,3}$ \\ Draft version September 30, 2018
}

\begin{abstract}
We present the analysis of the X-ray brightness and temperature profiles for six clusters belonging to both the Cool Core and Non Cool Core classes, in terms of the Supermodel (SM) developed by Cavaliere, Lapi \& Fusco-Femiano (2009). Based on the gravitational wells set by the dark matter halos, the SM straightforwardly expresses the equilibrium of the IntraCluster Plasma (ICP) modulated by the entropy deposited at the boundary by standing shocks from gravitational accretion, and injected at the center by outgoing blastwaves from mergers or from outbursts of Active Galactic Nuclei. The cluster set analyzed here highlights not only how simply the SM represents the main dichotomy Cool vs. Non Cool Core clusters in terms of a few ICP parameters governing the radial entropy run, but also how accurately it fits even complex brightness and temperature profiles. For Cool Core clusters like A2199 and A2597, the SM with a low level of central entropy straightforwardly yields the characteristic peaked profile of the temperature marked by a decline toward the center, without requiring currently strong radiative cooling and high mass deposition rates. Non Cool Core clusters like A1656 require instead a central entropy floor of a substantial level, and some like A2256 and even more A644 feature structured temperature profiles that also call for a definite floor extension; in such conditions the SM accurately fits the observations, and suggests that in these clusters the ICP has been just remolded by a merger event, in the way of a remnant cool core. The SM also predicts that dark matter halos with high concentration should correlate with flatter entropy profiles and steeper brightness in the outskirts; this is indeed the case with A1689, for which from $\mathrm{X}$ rays we find concentration values $c \sim 10$, the hallmark of an early halo formation. Thus we show the SM to constitute a fast tool not only to provide wide libraries of accurate fits to X-ray temperature and density profiles, but also to retrieve from the ICP archives specific information concerning the physical histories of dark matter and baryons in the inner and the outer cluster regions.

Subject headings: galaxies: clusters: general — galaxies: clusters: individual (A2199, A2597, A1689, A1656, A2256, A644) - X-rays: galaxies: clusters
\end{abstract}

\section{INTRODUCTION}

The evolution of a galaxy cluster comprises relaxed stretches punctuated by violent merger events affecting the gravitationally dominant dark matter (DM) halo, especially frequent in its early life after the initial collapse.

A number of such events may be caught in action, as in the extreme case of the Bullet Cluster (Markevitch et al. 2002; Clowe et al. 2006). As to the many quieter clusters, one may wonder how long and how precise a memory of similar if less dramatic events - and of the more frequent ones caused by central outbursts from Active Galactic Nuclei (AGNs) is retained by the hot diffuse baryons constituting their IntraCluster Plasma (ICP). We will show how a considerable amount of quantitative information may be retrieved even several Gyrs after the event from the X-ray bremsstrahlung emission by the ICP in the inner cluster regions.

On the other hand, the quiet stretches when external DM and gas are smoothly accreted across the virial radius $R$ will shape the outer DM density and gravitational potential, and hence the ICP distribution and X-ray bremsstrahlung emission in the cluster outskirts. Is there any simple and comprehensive, physical modeling conducive to a precise yet fast analysis of both these regions?

We will show how indeed our Supermodel (see Cavaliere, Lapi \& Fusco-Femiano 2009, thereafter CLFF09) enables

\footnotetext{
${ }^{1}$ INAF - Istituto di Astrofisica Spaziale e Fisica Cosmica, Via Fosso del Cavaliere, 00133 Roma, Italy.

${ }^{2}$ Dip. Fisica, Univ. 'Tor Vergata', Via Ricerca Scientifica 1, 00133 Roma, Italy.

${ }^{3}$ Astrophysics Sector, SISSA/ISAS, Via Beirut 2-4, 34151 Trieste, Italy.
}

us to accurately fit the full radial profiles of the X-ray observables, namely, the temperature $T(r)$ and the brightness $S(r) \propto n^{2}(r) T^{1 / 2}(r)$ that provides the baryon number density $n$. Whence we read out the imprints of the thermodynamic cluster history in the form of level, pattern, and time for depositions or injections of ICP specific entropy $k \equiv k_{B} T / n^{5 / 3}$ in Boltzmann units. Rather than relying on entropy profiles in the literature, we will focus on deriving the radial entropy runs from fitting the modeled primary observables for which precise, resolved and robust data (typically out to $R / 2$ ) exist as is the case for the six clusters considered here.

The Supermodel (SM) straightforwardly expresses the profiles $n(r)$ and $T(r)$ in the entropy-modulated equilibrium of the ICP within the potential wells provided by the dominant DM. These two components are related not only by their common containing well, but also by parallel accretion of surrounding DM and baryons into the cluster volume. Thus the SM tells the thermal and dynamical past of a cluster back to its formation time, to include subsequent mergers or AGN outbursts and current outer accretion.

The SM is built upon the physical DM distributions derived by Lapi \& Cavaliere (2009a) from the Jeans equation. These base on the radial run of the functional $K \equiv \sigma^{2} / \rho^{2 / 3}$, namely the 'DM entropy', that combines the 1-D velocity dispersion $\sigma(r)$ with the density $\rho(r)$. From $N$-body simulations $K(r)$ is found to constitute a truly universal halo feature as it always follows a powerlaw run $K(r) \propto r^{\alpha}$ throughout the halos' bulk (Taylor \& Navarro 2001; Dehnen \& McLaughlin 2005; Hoffman et al. 2007; Vass et al. 2009; Navarro et al. 2009). On the other hand, Lapi \& Cavaliere (2009a) compute $\alpha$, and find it 
to lie in the range $1.25-1.3$ from galaxies to massive clusters, narrowed down to $1.27-1.3$ from poor (overall mass $M \sim$ a few $\left.10^{14} M_{\odot}\right)$ to very rich $\left(M \sim\right.$ a few $\left.10^{15} M_{\odot}\right)$ clusters. Two basic features of these ' $\alpha$-profiles' entering the SM frame are briefly recalled in Appendix A.

We stress that our treatment of the $\alpha$-profiles (see Lapi \& Cavaliere 2009a,b) agrees with recent numerical simulations (Zhao et al. 2003; Diemand et al. 2007; Wang \& White 2008) in picturing the DM halo formation as a two-stage development. The epoch $z_{t}$ ranging from 0.5 to 2 marks the halo's transition from the stage of fast violent collapse scarred by major merging events, to one of progressively smoother and slower accretion building up the outskirts with little body perturbation.

During the latter, the DM profiles develop an increasing concentration $c \equiv R / r_{-2}$, that actually measures the current outer extension out to the virial radius $R$, relative to the region inner to $r_{-2}$ where $\rho(r)$ is flatter than $r^{-2}$ (see CLFF09). Starting with values $c \approx 3.5$ at the transition, $c$ increases to current values $c \approx 3.5\left(1+z_{t}\right)$, to attain values $c \approx 10$ or more for the $10 \%$ fraction of rich clusters with a transition as early as $z_{t} \approx 1.5$ (see Lapi \& Cavaliere 2009b); thus $c$ measures the dynamic age $z_{t}$ of the cluster. Since $R$ is typically of order 2 Mpc, values $c \approx 5-10$ imply $r_{-2} \approx 100-200 \mathrm{kpc}$; in the following, we will refer to 'outskirts' for the region $r>r_{-2}$, and to 'inner body' for the region $r \lesssim r_{-2}$. Based on the SM, we will discuss in particular how the outer distribution of the ICP entropy relates to the DM concentration.

The SM describes the equilibrium of the ICP in the DM potential well, as we recall in $\S 2$; there we briefly recap the main features of the ICP description in the form of the SM as developed by CLFF09, with the addition of Eq. (8) that enables computing the total mass. Then in $\S 3$ we describe our analysis procedure based on the SM. In $\S 4$ we apply such a procedure to the data for a set of six clusters with diverse profiles of X-ray brightness and temperature. Finally, in $\S 5$ we discuss the specific physical information we extract from our analysis of the X-ray observations.

In our treatment we adopt a standard flat cosmology with normalized matter density $\Omega_{M}=0.27$, dark energy density $\Omega_{\Lambda}=0.73$ and Hubble constant $H_{0}=70 \mathrm{~km} \mathrm{~s}^{-1} \mathrm{Mpc}^{-1}$, except when comparing with data otherwise scaled.

\section{THE SUPERMODEL}

We concentrate first on DM halos close to self-gravitational equilibrium under smooth and slow accretion, past the fast collapse stage and after any residual violent mergers, as discussed in $\S 5$. Within these DM gravitational wells, the ICP approaches hydrostatic conditions after outgoing blastwaves and shocks driven by central mergers or AGN outbursts have subsided.

In such conditions, the ICP density is governed by the balance between the gravitational force and the gradient of the pressure $p=n k_{B} T / \mu$; with the latter expressed as $p \propto k n^{5 / 3}$ in terms of ICP entropy, the balance reads

$$
\frac{1}{\mu m_{p} n} \frac{d\left(k n^{5 / 3}\right)}{d r}=-\frac{G M(<r)}{r^{2}}
$$

where $\mu \approx 0.6$ is the mean molecular weight, $m_{p}$ is the proton mass, and $G$ is the gravitational constant.

Once the radial entropy run $k(r)$ is given as discussed below, the solution of this $1^{\text {st }}$ order differential equation allowed us (CLFF09) to write the profiles of the gas temperature and density in the form

$$
\bar{T}(\bar{r})=\bar{k}(\bar{r}) \bar{n}^{2 / 3}(\bar{r})=\bar{k}^{3 / 5}(\bar{r})\left[1+\frac{2}{5} b_{R} \int_{\bar{r}}^{1} \mathrm{~d} \bar{r}^{\prime} \frac{\bar{v}_{c}^{2}\left(\bar{r}^{\prime}\right)}{\bar{r}^{\prime}} \bar{k}^{-3 / 5}\left(\bar{r}^{\prime}\right)\right] .
$$

Here barred variables are normalized to their boundary value at $r=R$; the squared circular velocity $\bar{v}_{c}^{2}(\bar{r})=\bar{M}(<\bar{r}) / \bar{r}$ is taken from the $\alpha$-profiles with their weak dependence on $\alpha$, and is expressed in Appendix $\mathrm{A}$ in terms of hypergeometric functions; finally, we define $b_{R} \equiv \mu m_{p} v_{c}^{2}(R) / k_{B} T(R)$.

The latter incorporates the boundary condition required for solving Eq. (1). It is physically convenient to fix such a reference value at the virial radius $r \approx R$ rather than at the center, where $v_{c}^{2}(r)$ vanishes steeply (see Lapi \& Cavaliere 2009a) while the ICP is often affected by violent stochastic events such as mergers and AGN outbursts. Moreover, at $r \sim R$ closely universal accretion of external InterGalactic Medium (IGM) prevails for both Cool Core (CC) and Non Cool Core (NCC) clusters; this holds to lowest order, although the related conversion of infall kinetic into thermal energy may differ somewhat related to cluster age and preheating conditions, as discussed in $\S 3$ and 5 .

In fact, in the absence of substantial preheating in the IGM larger than some 1/2 keV per particle (see Lapi et al. 2005; McCarthy et al. 2008), the energy conversion takes place in strong accretion shocks that linger at the virial radius (see Tozzi \& Norman 2001; Lapi et al. 2005; Voit 2005), to imply

$$
b_{R}=\frac{3}{2 \Delta \phi_{R}}
$$

that takes on values around 2.5. These obtain from maximal conversion at a strong shock of the gravitational infall energy, that yields $k_{B} T_{R}=\frac{2}{3} \mu m_{p} \Delta \Phi_{R}$; this quantity involves the specific kinetic energy $2 v_{R}^{2} \Delta \phi_{R}$ gained by the IGM that starts from the turning point its free fall toward $R$ across the potential drop $\Delta \Phi_{R}=v_{R}^{2} \Delta \phi_{R}$ (see Lapi et al. 2005). The latter is provided by the DM $\alpha$-profiles, and is conveniently normalized to the related $v_{R}^{2} \equiv v_{c}^{2}(R)$; whence Eq. (3) follows.

We stress that $k_{B} T_{R}$ lowers when $\Delta \phi$ and the proportional infall kinetic energy are smaller owing to a large concentration $c$; e.g., a value $c \approx 10$ holding for a cluster with an early transition (see $\S 1$ ), in place of $c \approx 4$ holding for clusters with a recent transition, implies $\Delta \phi$ to decrease from 0.57 to 0.47 (see CLFF09). On the other hand, $k_{B} T_{R}$ also lowers when a high preheating level of the IGM weakens the shock and impairs the conversion efficiency as discussed by Lapi et al. (2005) and Voit (2005).

To obtain the ICP temperature and density profiles the full radial run $k(r)$ of the entropy is required. Its physical modeling is based upon the notions that entropy is eroded by radiative cooling on the timescale $t_{c} \approx 65\left(k_{B} T / 5 \mathrm{keV}\right)^{1 / 2}\left(n / 10^{-3} \mathrm{~cm}^{-3}\right)^{-1}$ Gyr (Sarazin 1988), whilst it is enhanced by shocks; at $r \approx R$ it is deposited by standing accretion shocks, and in the central region is injected by outbound blastwaves terminating in a shock, as are driven by supersonic outflows from AGN outbursts or head-on mergers.

In the vicinity of $r \approx R$ the powerlaw approximation $k \propto r^{a}$ always applies (see CLFF09) with boundary value

$$
a_{R}=2.4-0.47 b_{R}
$$

varying around 1.1. Note that after Eq. (3) the upper bound 
$a_{R}=45 / 19 \approx 2.4$ obtains for large $\Delta \phi$ as then $b_{R}$ tends to vanish; at the other end, $a_{R}$ decreases for weaker and weaker shocks corresponding to smaller $\Delta \phi$ and/or relatively stronger preheating levels. As no other major sources or sinks of entropy occur from the boundary at a few Mpcs down to the central $r \sim 10^{2} \mathrm{kpc}$, in the outer range the entropy is deposited and stratified during the stage of slow accretion; thus a powerlaw radial run is set with slope $a(r)$ that stays close to its boundary value $a_{R}$.

At the center, instead, entropy may be intermittently injected by shocks driven by mergers reaching down there (McCarthy et al. 2007; Balogh et al. 2007), and by powerful AGNs residing in the central massive galaxies (see Valageas \& Silk 1999; Wu et al. 2000; Scannapieco \& Oh 2004; Lapi et al. 2005) as observed and reviewed by McNamara \& Nulsen (2007) and Markevitch \& Vikhlinin (2007).

Thus the full entropy profile that combines central injections with outer stratification may be described by the simple parametric expression (see Voit 2005 and references therein)

$$
\bar{k}(\bar{r})=\bar{k}_{c}+\left(1-\bar{k}_{c}\right) \bar{r}^{a} ;
$$

in fact, this approaches a constant value at small radii, and smoothly goes into a powerlaw at large radii.

Entropy profiles similar to Eq. (5) have been recently reported by Cavagnolo et al. (2009) from an analysis of a Chandra archival sample comprising 239 clusters. In fact, most of them are well fit by a power law at large radii plus a constant value $k_{0} \gtrsim k_{c}$ at small radii, with a basically bimodal distribution peaked at $k_{0} \approx 20 \mathrm{keV} \mathrm{cm}{ }^{2}$ and at $k_{0} \approx 150 \mathrm{keV} \mathrm{cm}^{2}$.

On the other hand, some clusters show evidence of a sharper entropy floor (e.g., Pratt et al. 2005 for A2218; see also Fig. 5 in Cavagnolo et al. 2009) that we represent not only with a level $k_{c}$ but also with a definite extension $r_{f}$, so that the corresponding radial entropy run reads

$$
\bar{k}=\bar{k}_{c}
$$

for $\bar{r} \leq \bar{r}_{f}$, and as

$$
\bar{k}=\bar{k}_{c}+\left(1-\bar{k}_{c}\right)\left(\frac{\bar{r}-\bar{r}_{f}}{1-\overline{r_{f}}}\right)^{a}
$$

for $\bar{r}>\bar{r}_{f}$. The scale $r_{f}$ may be interpreted as the terminal radius just reached by an outbound blastwave driven by a violent energy input at the center (see Lapi et al. 2005); at $r_{f}$ its decreasing Mach number $\mathcal{M}(r)$ has decayed to unity and the blast has stalled and degraded into adiabatic sound waves, as caught in action by Fabian et al. (2006) in the Perseus Cluster (see also $\S 5$ for a discussion). In this picture, Eq. (5) represents a later stage caused by diffusive smoothing and mixing of such a radial imprint, while the enhanced entropy level is still high before radiative erosion has set in.

From the gas temperature and density profiles given by Eq. (2) we also derive the distribution of the gravitating mass in the form (see Sarazin 1988)

$$
M(<\bar{r}) \simeq 3.65 \times 10^{13}\left(\frac{k_{B} T_{R}}{\mathrm{keV}}\right)\left(\frac{R}{\mathrm{Mpc}}\right) b_{R} \bar{r} \bar{v}_{c}^{2}(\bar{r}) M_{\odot},
$$

where the quantity $\bar{r} \bar{v}_{c}^{2}(r)$ grows slowly with $\bar{r}$ and saturates to unity.

\section{SUPERMODEL ANALYSIS}

Here we describe in detail how we use the SM to account for the X-ray brightness and temperature profiles for both classes of CC and NCC clusters (Molendi \& Pizzolato 2001; Leccardi \& Molendi 2009); in particular, we will examine in detail the following six clusters: A2199, A2597, A1689, A1656, A2256, A644. These have been collected from the literature on the basis of the quality and detail of the X-ray data, keeping a balance between the $\mathrm{CC}$ and NCC classes. We fit with the SM the profiles of emission weighted temperature and X-ray brightness, as given by Eqs. (9) and (10) below. The parameter values are pinned down on using a standard $\chi^{2}$ minimization procedure, and the uncertainties are quoted at the $68 \%$ confidence level.

The free parameters are set as follows. The DM halo distributions depend weakly on the index $\alpha$ that we fix at the value $\alpha \approx 1.27$, and are strongly marked by the concentration $c$ that we leave as a free parameter. The ICP profiles are parameterized by the slope $a$ and the central value $k_{c}$ that define the radial entropy run after Eq. (5); in some clusters an acceptable fit requires to introduce the size $r_{f}$ of the central floor after Eqs. (6) and (7).

In the SM as with all models the virial radius $R$ intervenes to set the data distance scale, as well as the bound to l.o.s. integrations; we keep it fixed when a robust determination is available from the literature, e.g., from observations of galaxy dynamics, or 'red sequence' termination, or gravitational lensing. Otherwise, we determine it from fitting (in the range where the data are reliable) the projected profile of the emission-weighted temperature

$$
\left\langle k_{B} T(\bar{w})\right\rangle=k_{B} T_{R} \frac{\int_{0}^{\sqrt{1-\bar{w}^{2}}} \mathrm{~d} \bar{\ell} \bar{n}^{2}(\bar{r}) \Lambda[T(\bar{r})] \bar{T}(\bar{r})}{\int_{0}^{\sqrt{1-\bar{w}^{2}}} \mathrm{~d} \bar{\ell} \bar{n}^{2}(\bar{r}) \Lambda[T(\bar{r})]},
$$

in terms of the projected radius $\bar{w} \equiv w / R$. In the following we approximate the detailed cooling function with $\Lambda(T) \propto$ $T^{1 / 2}$ as appropriate for the ICP in hot clusters. The above relation not only pins down the (horizontal) scale $R$, but also sets the normalization (vertical) scale $T_{R}$. The knowledge of $R$ and $T_{R}$ allows us to derive the ICP density $n_{R}$ from fitting the brightness distribution

$$
\begin{aligned}
S(\bar{w}) & \approx S_{0}\left(\frac{R}{\mathrm{Mpc}}\right)\left(\frac{n_{R}}{10^{-3} \mathrm{~cm}^{-3}}\right)^{2}\left(\frac{k_{B} T_{R}}{\mathrm{keV}}\right)^{1 / 2} \times \\
& \times \int_{0}^{\sqrt{1-\bar{w}^{2}}} \mathrm{~d} \bar{\ell} \bar{n}^{2}(\bar{r}) \bar{T}^{1 / 2}(\bar{r}) F\left[E_{1}, E_{2}, T(\bar{r})\right] ;
\end{aligned}
$$

here $S_{0} \approx 3.4 \times 10^{-13}(1+z)^{-4} \mathrm{erg} \mathrm{s}^{-1} \mathrm{~cm}^{-2} \operatorname{arcmin}^{-2}$, and the factor $F\left(E_{1}, E_{2}, T\right) \simeq e^{-E_{1} / k_{B} T}-e^{-E_{2} / k_{B} T}$ takes into account specific instrumental bands $E_{2}-E_{1}$ (e.g., Ettori 2000).

The SM actually predicts the values of $T_{R}, n_{R}$ and $k_{R}=$ $k_{B} T_{R} / n_{R}^{2 / 3}$ from extrapolating the profiles into the outer cluster regions; observing the latter challenges the sensitivity and defies the resolution of most current instruments, but will constitute a main target for the next-generation X-ray telescopes planned to study low surface brightness plasmas (see $\S 5$ ).

With the use of such facilities $R$ will be read out from the profiles as the position of the shock discontinuities in $n(r)$ and $T(r)$ above the values prevailing in the IGM (see Lapi et al. 2005), even though such discontinuities may be blended by complex texture of the shocks and smoothed by projection integrations (see Tormen et al. 2004). On the other hand, substantial preheating of the IGM may weaken the accretion shocks and lower $T_{R}$. Such conditions will be indicated by any 

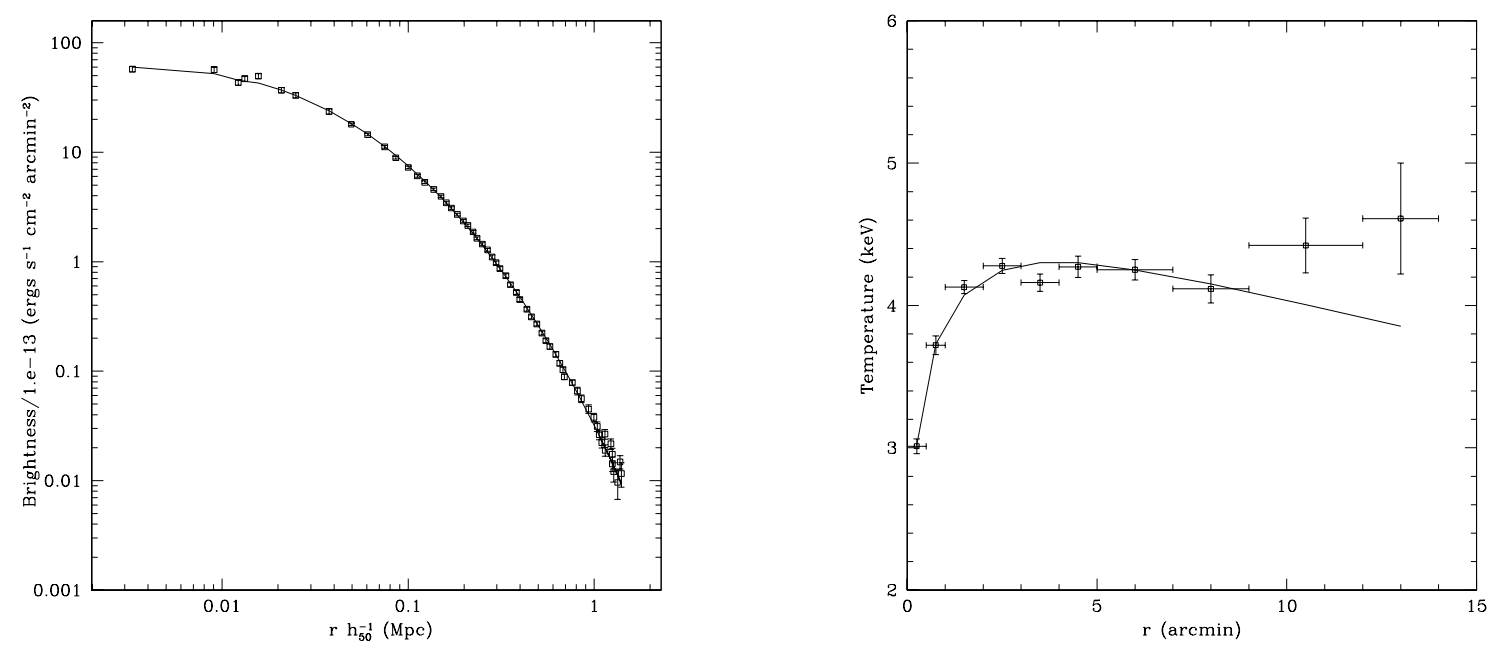

FIG. 1.- Abell 2199. Left panel: The solid line is our fit $\left(\chi^{2}=73.9 / 56\right)$ to the brightness profile measured by Mohr et al. (1999), on adopting the entropy profile given by Eq. (5). Right panel: The solid line is our fit $\left(\chi^{2}=15.8 / 5\right)$ to the radial temperature profile measured by Snowden et al. (2008), on adopting the entropy profile given by Eq. (5).

discrepancy between the value resulting from Eq. (9) and the reference value $k_{B} T_{R}$ corresponding to strong shocks in a halo of given concentration.

\section{RESULTS FOR INDIVIDUAL CLUSTERS}

Next we present the results for each of our six clusters; the individual fitting parameters are given in the corresponding subsections and are collected in Table 1.

\subsection{Abell 2199}

A2199 is a rich cluster at $z \approx 0.031$ centered on the $\mathrm{cD}$ galaxy NGC6166 that hosts the radio source 3C338. The latter shows evidence of several events of radio emission, as witnessed by the clear correspondence between positions of radio lobes and depressed X-ray surface brightness ('cavities').

This is a CC cluster with the temperature profile declining from about $200 \mathrm{kpc}$ toward the center as observed with Chandra by Johnstone et al. (2002) and with XMM-Newton by Snowden et al. (2008). Fig. 1 shows the SM fits to the projected emission-weighted temperature profile given by the latter authors, and to the brightness distribution by Mohr et al. (1999) obtained with ROSAT. Both profiles do not show evidence of an entropy floor down to $r \approx 2 \mathrm{kpc}$.

From the SM, we obtain the virial radius $R=2.1_{-0.4}^{+0.1} \mathrm{Mpc}$ and the halo concentration parameter $c=6.7_{-1.0}^{+1.0}$. In the inner ICP regions we find $\bar{k}_{c}=0.39_{-0.16}^{+0.16} \times 10^{-2}$. Throughout the cluster body we derive the entropy slope $a=0.95_{-0.01}^{+0.01}$. In the outskirts we find $k_{B} T_{R}=1.93_{-0.05}^{+0.05} \mathrm{keV}$ and $n_{R}=1.40_{-0.03}^{+0.03} \times$ $10^{-5} \mathrm{~cm}^{-3}$, yielding $k_{R}=3320_{-130}^{+130} \mathrm{keV} \mathrm{cm}^{2}$. Correspondingly, we obtain $k_{c}=13_{-6}^{+6} \mathrm{keV} \mathrm{cm}^{2}$.

\subsection{Abell 2597}

$\mathrm{A} 2597$ is a nearby cluster at $z \approx 0.08$ that harbors the central radio source PKS 2322-123; it shows features in different spectral regions. In particular, Chandra X-ray observations show a bright central region and two low surface brightness ghost cavities (McNamara et al. 2001), adding to other core structures suggestive of inner cavities (Clarke et al. 2005). A2597 is a CC cluster as is also confirmed with
XMM-Newton observations by Morris \& Fabian (2005) and Snowden et al. (2008). The average temperature is around 2.6 $\mathrm{keV}$ (Sarazin \& McNamara 1997).

We fit (see Fig. 2) the surface brightness data obtained by ROSAT/PSPC observations (Xue \& Wu 2000) and the temperature profile observed by XMM-Newton (Snowden et al. 2008), on considering the two entropy profiles given by Eqs. (5) or by Eqs. (6) and (7). The latter include the additional parameter $r_{f}=50_{-7}^{+7} \mathrm{kpc}$ that turns out to be significant at the $99.97 \%$ level after the $F$-test, and may be interpreted as the effect of the central radio source PKS2322-123. On the other hand, the size of the entropy floor lies within the first bin of the temperature data (see Fig. 3), below the resolution of our profile.

From the SM, we obtain the virial radius $R=1.9_{-0.4}^{+0.4} \mathrm{Mpc}$ and the halo concentration parameter $c=7.2_{-5.2}^{+5.0}$. In the inner ICP regions we find $\bar{k}_{c}=0.21_{-0.12}^{+0.48} \times 10^{-2}$. Throughout the cluster body we derive the entropy slope $a=0.71_{-0.05}^{+0.05}$. In the outskirts we obtain $k_{B} T_{R}=2.1_{-0.6}^{+0.8} \mathrm{keV}$ and $n_{R}=2.0_{-0.2}^{+0.2} \times 10^{-5}$ $\mathrm{cm}^{-3}$, yielding $k_{R}=2850_{-940}^{+1370} \mathrm{keV} \mathrm{cm}^{2}$. Correspondingly, we obtain $k_{c}=6_{-4}^{+18} \mathrm{keV} \mathrm{cm}^{2}$.

\subsection{Abell 1689}

A1689 is a rich cluster at $z \approx 0.183$ that has been studied in various spectral regions; a joint analysis of HST/ACS and Chandra measurements has been carried out by Lemze et al. (2008) to derive the profiles of the ICP density and temperature. The cluster is centered on a cD galaxy, is roughly spherical and appears fairly relaxed (but see Andersson \& Madejski 2004). The $X$-ray luminosity is $L_{X} \approx 10^{45} \mathrm{erg} \mathrm{s}^{-1}$ in the energy band $0.5-7 \mathrm{keV}$, due to the hot ICP with $k_{B} T \approx 9.4 \mathrm{keV}$ (Ebeling et al. 1996; Xue \& Wu 2002; Andersson \& Madejski 2004). Strong and weak lensing observations (see Lemze et al. 2008 and references therein) show the projected mass profile to continuously flatten toward the center, with a steep outer profile that require a high concentration $c \approx 13.7_{-1.1}^{+1.4}$ (see Broadhurst et al. 2008 and references therein), the hallmark of an early transition.

With the SM we have fitted the ICP temperature profile observed with XMM-Newton by Snowden et al. (2008), and the 

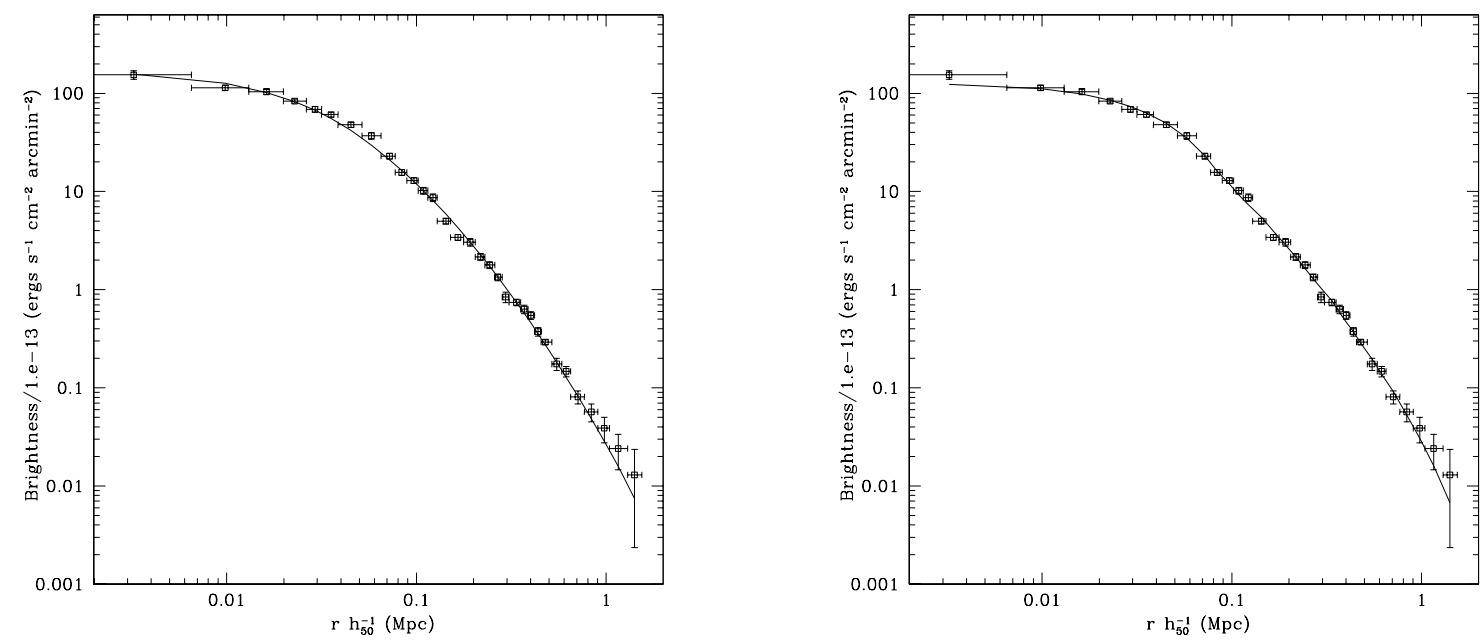

FIG. 2.- A2597. Left panel: The solid line is our fit $\left(\chi^{2}=53.5 / 28\right)$ to the brightness profile measured by Xue \& Wu (2000), on adopting the entropy profile given by Eq. (5). Right panel: The solid line is our fit $\left(\chi^{2}=32.3 / 27\right)$ on adopting the entropy profile given by Eqs. (6) and (7).

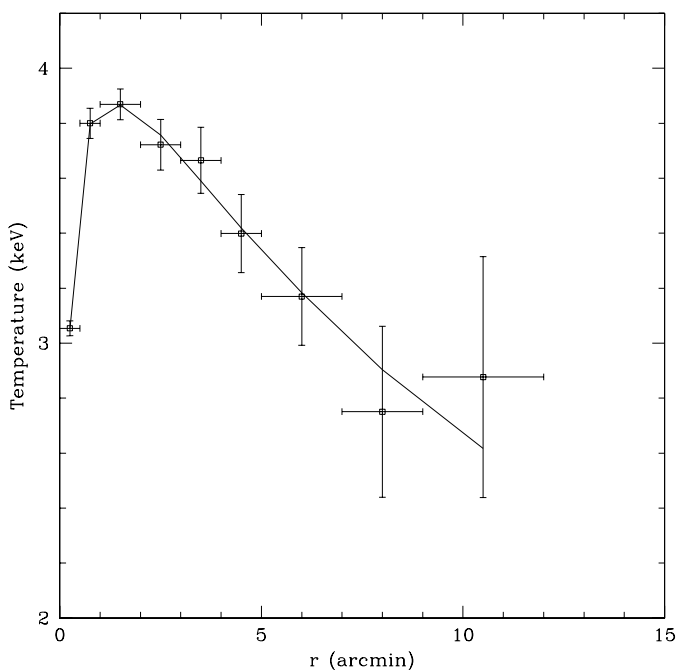

FIG. 3.- A2597. The solid line is our fit $\left(\chi^{2}=1.1 / 4\right)$ to the temperature profile measured by Snowden et al. (2008), on adopting the entropy profile given by Eq. (5).

surface brightness profile observed by Mohr et al. (1999) and by Lemze et al. (2008). In agreement with the latter authors, our fits (see Fig. 4) do not require an entropy floor; any central flattening may affect only radii $r \lesssim 2 \times 10^{-3} R \approx 4 \mathrm{kpc}$.

We adopt the virial radius $R=2.1 \mathrm{Mpc}$ fixed at the value given by Lemze et al. (2008), and from the SM we find the halo concentration parameter $c=13.6_{-4.3}^{+4.3}$. In the inner ICP regions we find $\bar{k}_{c} \approx 2.4_{-0.8}^{+0.8} \times 10^{-2}$. Throughout the cluster body we derive the low empirical value of the entropy slope $a=0.80_{-0.06}^{+0.06}$ (consistent with the value $0.82_{-0.02}^{+0.02}$ measured by Lemze et al. 2008), that we relate after Eqs. (3) and (4) to the high concentration. In the outskirts we obtain $k_{B} T_{R}=4.4_{-0.6}^{+0.6}$ $\mathrm{keV}$ and $n_{R}=3.2_{-0.1}^{+0.1} \times 10^{-5} \mathrm{~cm}^{-3}$, yielding $k_{R} \approx 4360_{-590}^{+590} \mathrm{keV}$ $\mathrm{cm}^{2}$. Correspondingly, we obtain $k_{c}=105_{-49}^{+49} \mathrm{keV} \mathrm{cm}^{2}$.

Finally, in Fig. 5 we show the mass profile derived from the SM after Eq. (8) on using the parameters obtained from our fits; this turns out to be in good agreement with the total mass profile obtained from the lensing data by Lemze et al. (2008).

\subsection{Abell 1656 (Coma Cluster)}

A1656, the popular Coma Cluster, is a very rich cluster at $z \approx 0.023$. Its ICP at temperature $k_{B} T \approx 8 \mathrm{keV}$ is a powerful X-ray emitter with luminosity $L_{X} \sim 10^{45} \mathrm{erg} \mathrm{s}^{-1}$ (see David et al. 1993) extending out to about $1^{\circ}$ from the center (e.g., Briel et al. 2001).

Several determinations of the virial radius ranging between 2 and $3 \mathrm{Mpc}$ are given in the literature (Castander et al. 2001; Łokas \& Mamon 2003; Kubo et al. 2007; Gavazzi et al. 2009). For our analysis, we have adopted the value of 2.2 Mpc reported by Gavazzi et al. (2009), and checked that our results depend only weakly on this choice within 1 standard deviation.

From the SM we find the halo concentration parameter $c=3.0_{-0.8}^{+0.8}$, the hallmark of a recent formation (see $\S 1$ ). In the inner ICP regions we find $\bar{k}_{c} \approx 10_{-1}^{+1} \times 10^{-2}$. Throughout the cluster body we derive the entropy slope $a=1.30_{-0.23}^{+0.46}$. In the outskirts we obtain $k_{B} T_{R}=5.7_{-1.0}^{+1.0} \mathrm{keV}$ and $n_{R}=3.66_{-0.15}^{+0.15} \times$ $10^{-5} \mathrm{~cm}^{-3}$, yielding $k_{R}=5170_{-1045}^{+1060} \mathrm{keV} \mathrm{cm}{ }^{2}$. Correspondingly, we find $k_{c}=520_{-160}^{+160} \mathrm{keV} \mathrm{cm}^{2}$.

Our fits to the the brightness data observed with ROSAT by Mohr et al. (1999) are obtained on using the two entropy profiles given by Eqs. (5) or by Eqs. (6) and (7), and are illustrated in Fig. 6; the $\chi^{2}$ values in the caption strongly indicate the presence of an entropy floor with extension $r_{f}=250_{-74}^{+44}$ kpc. On the other hand, the fit to the emission-weighted temperature profile (see Fig. 7) obtained from XMM-Newton observations by Snowden et al. (2008) is roughly isothermal, and within its resolution does not require by itself an entropy floor.

All the above concurs to a picture of A1656 as a halo that has just collapsed and undergone major mergers, as confirmed by the structured features around the main galaxies NGC4874 and NGC4889 and by the ongoing fall of the NGC4839 galaxy group into the main body of the cluster (Adami et al. 2005). A1656 also exhibits extended radio emissions, including a young radio halo close to center and an outer radio relic located in the SW direction beyond the NGC4839 galaxy group (Giovannini et al. 1991, and references therein). 

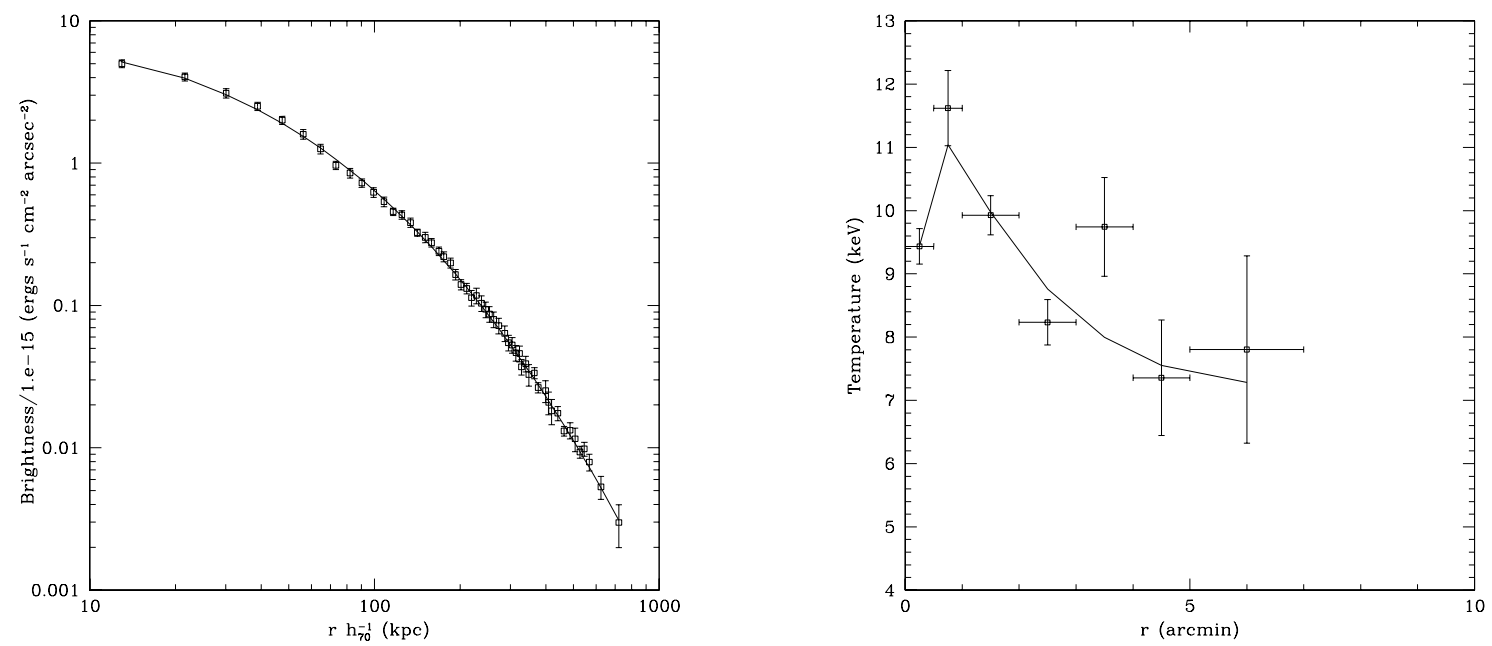

FIG. 4.- A1689. Left panel: The solid line is our fit $\left(\chi^{2}=19.8 / 49\right)$ to the brightness profile measured by Lemze et al. (2008), on adopting the entropy profile given by Eq. (5). Right panel: The solid line is our fit $\left(\chi^{2}=9.2 / 2\right)$ to the temperature profile measured by Snowden et al. (2008), on adopting the entropy profile given by Eq. (5).

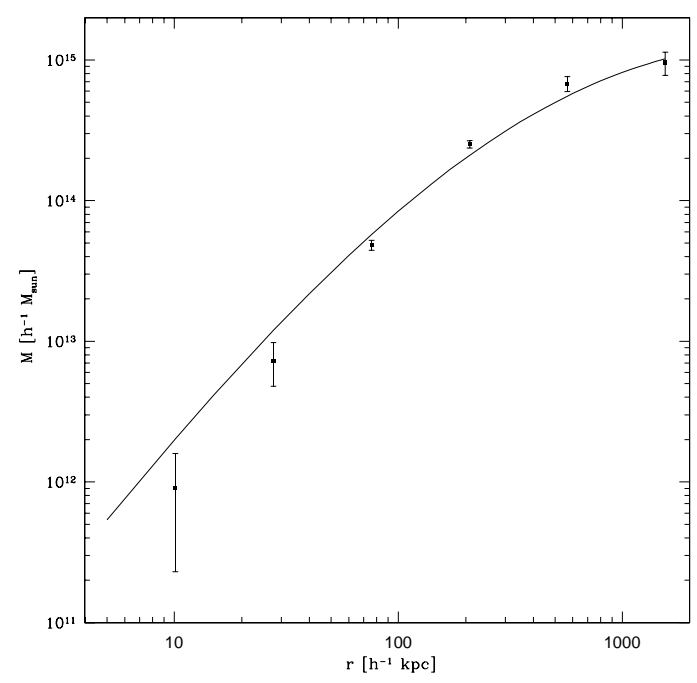

FIG. 5.- A1689. The solid line is our fit to the mass profile measured by Lemze et al. (2008) through gravitational lensing observations.

From Eq. (8) we derive an overall mass $M_{R}=1.24_{-0.66}^{+0.44} \times$ $10^{15} M_{\odot}$, in agreement with the value of $9.7_{-3.5}^{+6.1} \times 10^{14} M_{\odot}$ obtained by Gavazzi et al. (2009) from gravitational lensing.

\subsection{Abell 2256}

A2256 at $z \approx 0.06$ is a complex cluster, as highlighted by several studies in various spectral bands; it is a strong Xray emitter with luminosity $L_{X} \approx 10^{45} \mathrm{erg} \mathrm{s}^{-1}$. We have used the SM to fit the ROSAT brightness distribution by Mohr et al. (1999) and the temperature profile observed with XMMNewton by Snowden et al. (2008), on using the entropy profiles given by Eqs. (5) or by Eqs. (6) and (7); our results are illustrated in Fig. 8 and 9.

From the SM, we find the virial radius $R=2.2_{-0.3}^{+0.3} \mathrm{Mpc}$, and the halo concentration parameter $c=2.7^{+1.7}$. In the inner ICP regions we find $\bar{k}_{c}=6.2_{-3.1}^{+3.9} \times 10^{-2}$. Throughout the cluster body we derive the entropy slope $a=1.48_{-0.29}^{+0.35}$. In the outskirts we obtain $k_{B} T_{R}=4.4_{-0.9}^{+0.9} \mathrm{keV}$ and $n_{R}=3.64_{-0.22}^{+0.04} \times 10^{-5} \mathrm{~cm}^{-3}$, yielding $k_{R}=4000_{-980}^{+1097} \mathrm{keV} \mathrm{cm}^{2}$. Correspondingly, we find $k_{c}=248_{-185}^{+224} \mathrm{keV} \mathrm{cm}^{2}$.

Both the brightness and temperature distributions call for an entropy floor; basing on Eqs. (6) and (7) we derive a floor radius $r_{f}=264_{-80}^{+102} \mathrm{kpc}$ from the brightness, an evidence reinforced by the value $r_{f}=265_{-170}^{+80} \mathrm{kpc}$ we obtain from the temperature profile. Its introduction allows the SM to fit well the structured temperature profile of A2256; this features a temperature decrement similar to a CC cluster (e.g., Piffaretti et al. 2005; Leccardi \& Molendi 2009), but at small radii $T(r)$ reverses its trend and increases toward the center. Such a behavior is understood from the relation $T(r) \propto k(r) n(r)^{2 / 3}$; in the inner region where the entropy is constant, the temperature is expected to decrease outwards following the density, while for $r>r_{f}$ the entropy starts to increase and to dominate the density decrement, so raising the temperature out to a peak at $r \approx 350 \mathrm{kpc}$ (about $5^{\prime}$ ); beyond the peak the density steepens and offsets the entropy rise. The central temperature behavior suggests that the energy delivered by a merger has remolded the whole inner structure, and hence that the ICP is itself thermodynamically young within a dynamically young DM halo.

The halo's young age is supported by the low values $c \approx 4$ of the DM concentration, as expected in clusters with a recent transition from fast collapse to slow accretion (see $\S 1$ ). It is also indicated by the recent and intense merger activity that characterize A2256, as pinned down by ROSAT, Chandra and XMM-Newton observations (Briel et al. 1991; Sun et al. 2002; Berrington et al. 2002; Miller et al. 2003; Bourdin \& Mazzotta 2008). Such a merger activity is quite recent as indicated by the presence of relativistic electrons with lifetime of $10^{-1}$ Gyr that enlighten the radio halo and the very extended, bright relic in the NW region of the cluster (Bridle \& Formalont 1976; Kim 1999; Clarke \& Ensslin 2006). All that concurs with the high central entropy level pinned down by the SM analysis. 

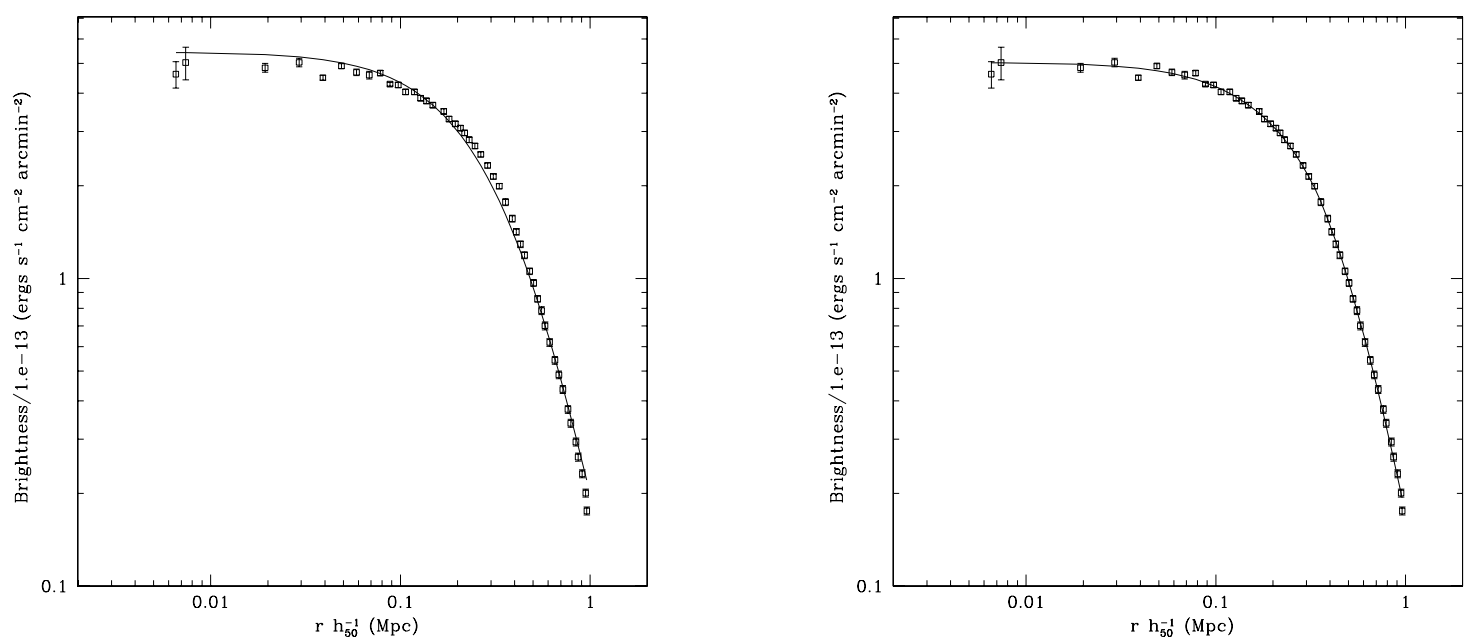

FIG. 6.- A1656. Left panel: The solid line is our fit $\left(\chi^{2}=408.5 / 44\right)$ to the brightness profile measured by Mohr et al. (1999), on adopting the entropy profile given by Eq. (5). Right panel: The solid line is our fit $\left(\chi^{2}=53.0 / 43\right)$ on adopting the entropy profile given by Eqs. (6) and (7).
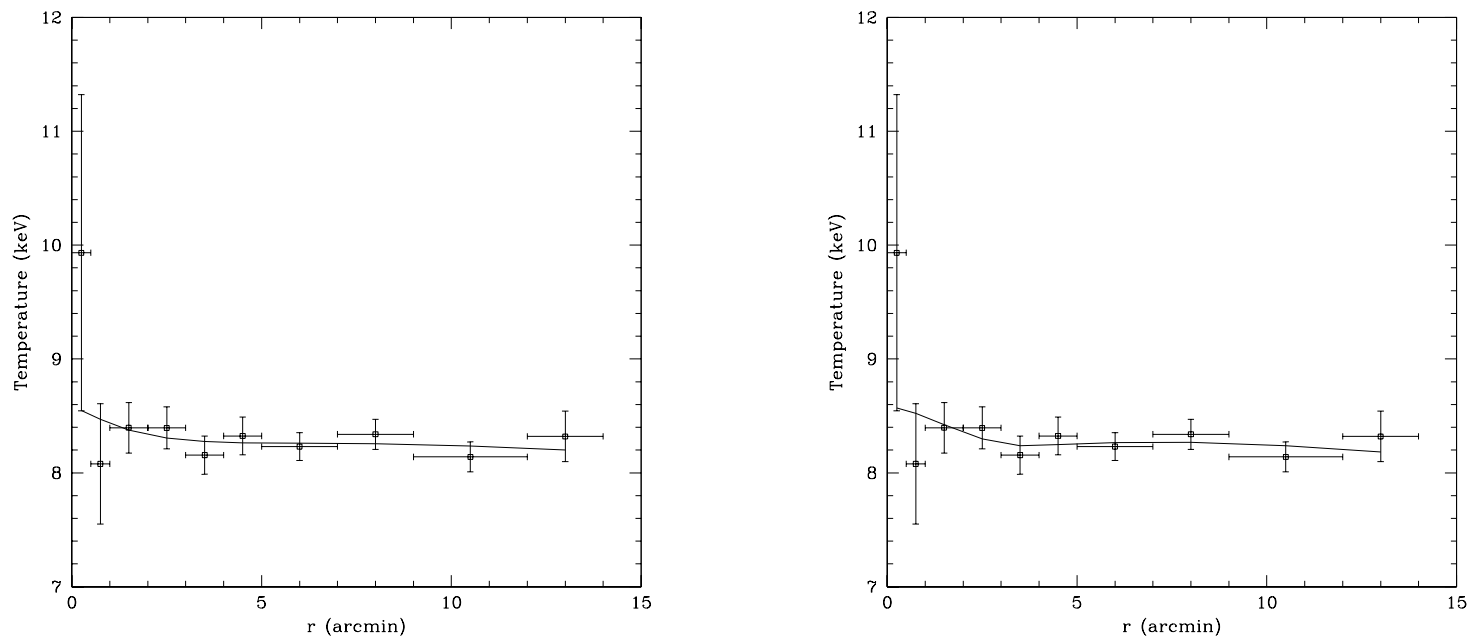

FIG. 7.- A1656. Left panel: The solid line is our fit $\left(\chi^{2}=3.7 / 6\right)$ to the temperature profile measured by Snowden et al. (2008), on adopting the entropy profile given by Eq. (5). Right panel: The solid line is our fit $\left(\chi^{2}=3.7 / 5\right)$ on adopting the entropy profile given by Eqs. (6) and (7).

A644 is a radio quiet cluster at $z \approx 0.07$. The $\mathrm{X}$-ray imaging with Chandra by Buote et al. (2005) shows that, while the outer ICP is smooth and relaxed, the central $\sim 10^{2} \mathrm{kpc}$ features very complex and interesting conditions. Although the latter do not bear strong marks of past radio activity like X-ray cavities or filaments, the peak of the X-ray emission is found to be shifted by about $60 \mathrm{kpc}$ from the cluster centroid (see also Bauer \& Sarazin 2000), beyond the cD galaxy located at about $40 \mathrm{kpc}$. The temperature profiles differ when centered on the centroid or on the X-ray peak; the former profiles deviates only weakly from a constant, while the latter shows a behavior similar to, if sharper than A2256, in that it decreases considerably toward the center before reversing its course and rising again at small radii (Buote et al. 2005). Our SM fits to this temperature profile, obtained on using Eq. (5) or Eqs. (6) and (7), are illustrated in Fig. 10; the fit to the brightness profile centered on the cluster centroid is illustrated in Fig. 11.

We find the virial radius $R=2.1_{-0.4}^{+0.4} \mathrm{Mpc}$ and the halo concentration parameter $c=3.9-0.2$. At the center we find $\bar{k}_{c}=0.7_{-0.1}^{+0.1} \times 10^{-2}$. On using Eqs. (6) and (7) we derive a radius $r_{f}=61_{-41}^{+36} \mathrm{kpc}$, whose need is substantiated by the $F$-test at $99.9 \%$ level and also strengthened by the fit to the Chandra brightness profile centered on the X-ray peak (D. Buote, private communication) that consistently yields $r_{f}=66_{-9}^{+8} \mathrm{kpc}$. Throughout the cluster body we derive the entropy slope $a=1.06_{-0.11}^{+0.11}$. In the outskirts we find $k_{B} T_{R}=4.9_{-1.8}^{+1.8} \mathrm{keV}$ and $n_{R}=2.88_{-0.19}^{+0.19} \times 10^{-5} \mathrm{~cm}^{-3}$, yielding $k_{R}=5200_{-2140}^{+2140} \mathrm{keV} \mathrm{cm}^{2}$. Correspondingly, we find $k_{c}=36_{-20}^{+20} \mathrm{keV} \mathrm{cm}^{2}$. Finally, from Eq. (8) we derive the total mass $M=1.2_{-0.7}^{+0.7} \times 10^{15} M_{\odot}$.

Note that if one insisted on applying the SM also to temperature and brightness profiles from the centroid on the basis of Eqs. (6) and (7), one would obtain $r_{f}=104_{-4}^{+4} \mathrm{kpc}$ and a related lower bound $k_{c} \approx 124^{+120} \mathrm{keV} \mathrm{cm}^{2}$. The large variance in the above parameters entering the entropy floors signals complex substructures, that may be interpreted as a high density, low entropy clump ('cold drop') around the X-ray peak. Our results agrees with the analysis by Buote et al. (2005), who 

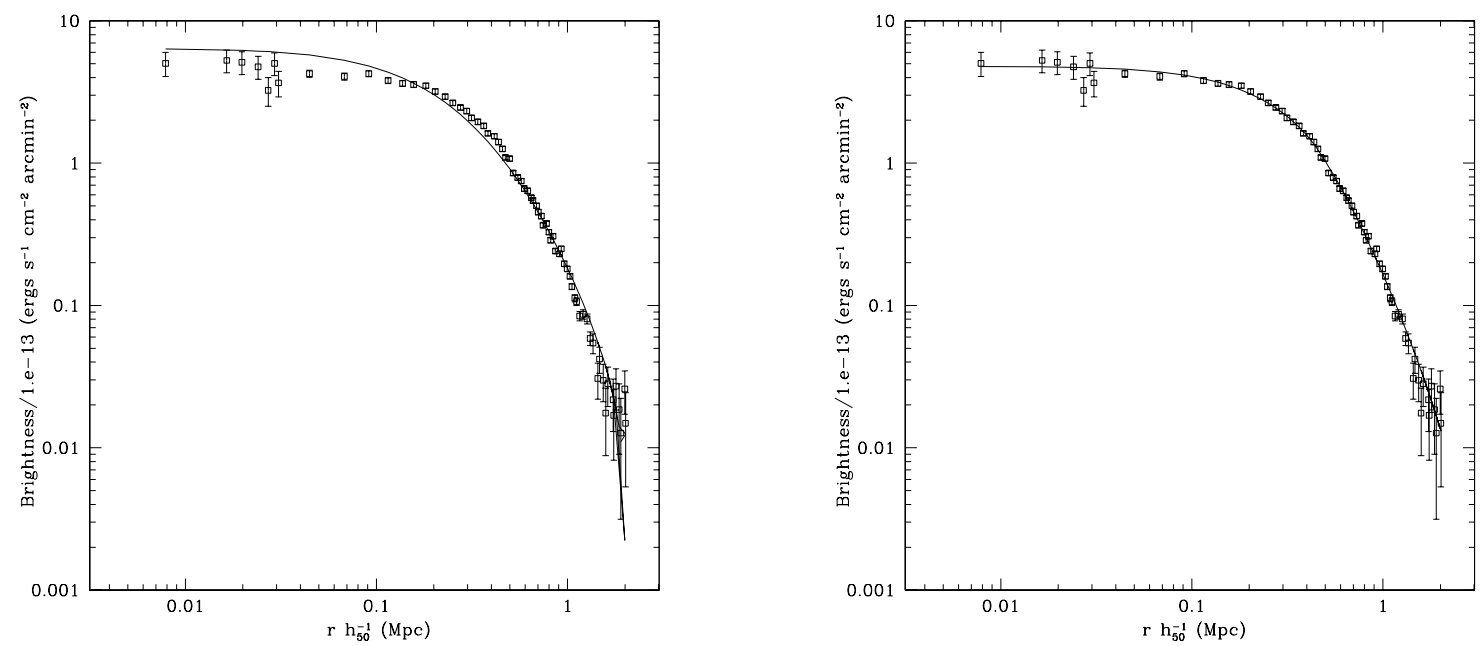

FIG. 8.- A2256. Left panel: The solid line is our fit $\left(\chi^{2}=434.1 / 65\right)$ to the brightness distribution measured by Mohr et al. (1999), on adopting the entropy profile given by Eq. (5). Right panel: The solid line is our fit $\left(\chi^{2}=109.7 / 64\right)$ on adopting the entropy profile given by Eqs. (6) and (7).
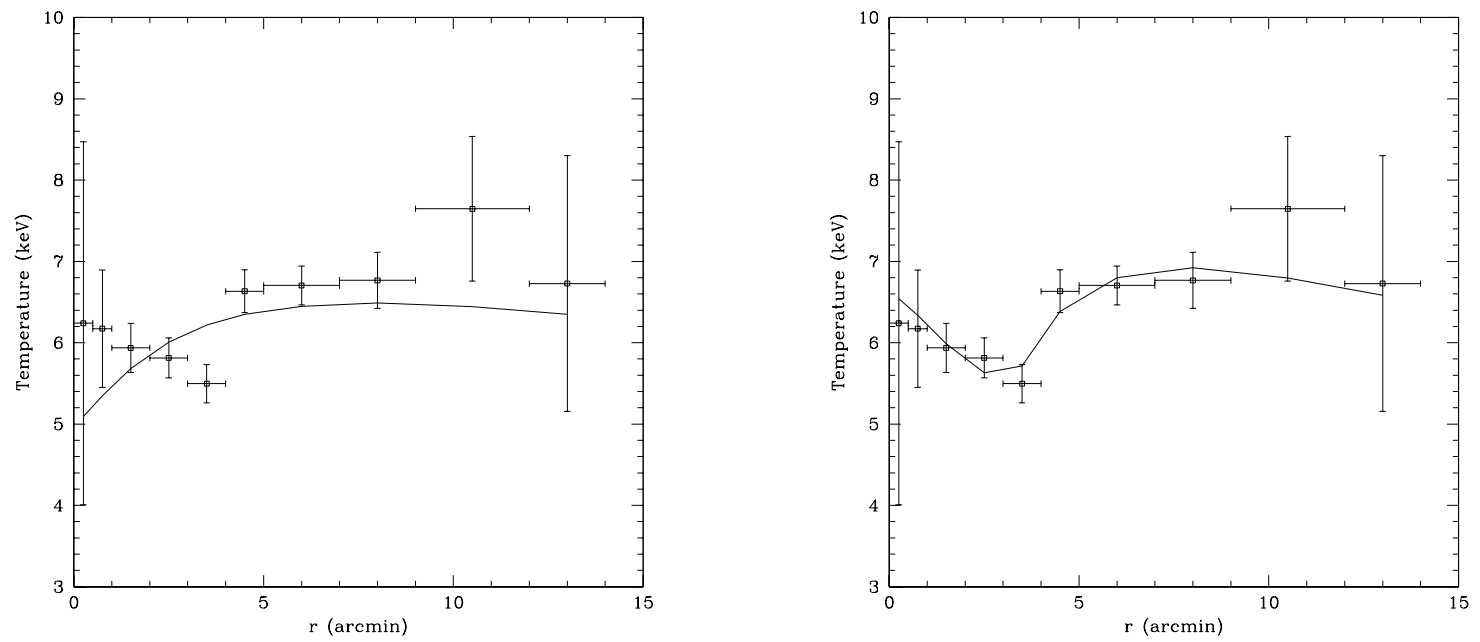

FIG. 9.- A2256. Left panel: The solid line is our fit $\left(\chi^{2}=12.4 / 5\right)$ to the temperature profile measured by Snowden et al. (2008), on adopting the entropy profile given by Eq. (5). Right panel: The solid line is our fit $\left(\chi^{2}=3.6 / 4\right)$ on adopting the entropy profile given by Eqs. (6) and (7). Note that the first bin covers the range out to about $0.05 h_{50}^{-1} \mathrm{Mpc}$ in Fig. 8.

in terms of two differently centered $\beta$-models (Cavaliere \& Fusco-Femiano 1976) find two core sizes similar to our extensions $r_{f}$.

In view of the lack of radio emission and X-ray cavities, this complexity may be understood in terms of a merger having just remolded an inner region of the ICP (see Henning et al. 2009). We stress that such ICP substructures constitute a common, but progressively more pronounced trait, of A1656, A2256 and A644.

\section{DISCUSSION AND CONCLUSIONS}

In this paper we have analyzed with the Supermodel (SM) the profiles of X-ray temperature and surface brightness of the IntraCluster Plasma (ICP) in a set of six clusters (adding to the three ones preliminarily reported in CLFF09) with existing detailed data. We have shown how effective is our SM to represent and understand the main Cool Core /Non Cool Core dichotomy in terms of two physical parameters marking the full ICP entropy profile: the central value $k_{c}$, and the outer slope $a$ (see Figs. 1-7). Moreover, the SM makes sense of more structured profiles (see Figs. 8-11) in terms of the additional, physical parameter $r_{f}$ marking the extension of the entropy floor.

The working of the SM may be reduced to the bones as follows. The spatial scale for the temperature peak in the $\mathrm{CCs}$, and for the outward temperature and density declines in all clusters, is set by the underlying gravitationally dominant DM distribution; specifically, such a scale is set by the peak of the DM velocity dispersion $\sigma^{2}(r)$ at the position $r_{m} \approx r_{-2}$, that divides the 'inner' from the 'outer' cluster regions (see Figs. 1 and 3 of CLFF09). In fact, the SM ensures that the approximation $T \simeq \sigma^{2} / \beta_{m}$ [with $\beta_{m} \equiv \mu m_{p} \sigma^{2}\left(r_{m}\right) / k_{B} T\left(r_{m}\right)$, see CLFF09] is to hold closely for all clusters around $r_{m}$, and over a wide radial range for the $\mathrm{CCs}^{4}$. In the inner ICP regions, the temperature and density profiles are governed primarily by the

\footnotetext{
${ }^{4}$ The formal reason why around $r_{m}$ the temperature $T(r)$ mirrors so well the DM dispersion $\sigma^{2}(r)$ is that the latter can be expressed quite similarly to
} 

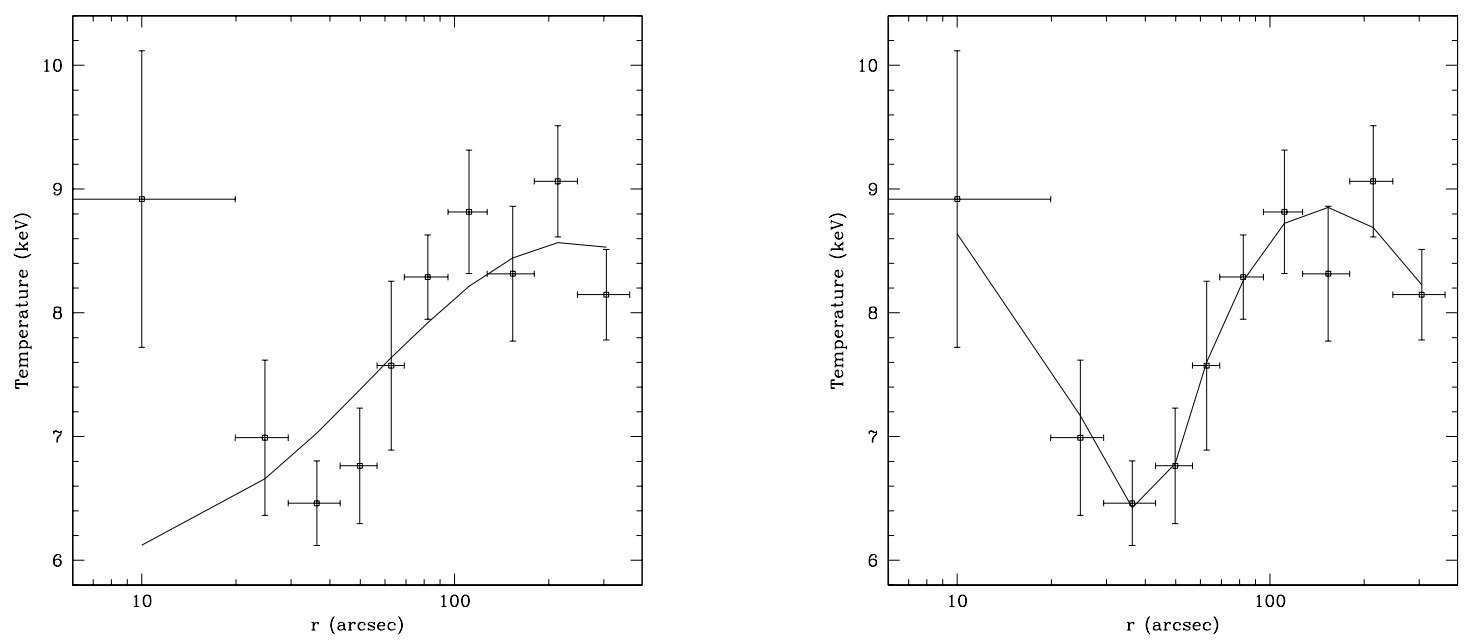

FIG. 10.- A644. Left panel: The solid line is our fit $\left(\chi^{2}=15.2 / 5\right)$ to the temperature profile centered on the X-ray peak measured by Buote et al. (2005), on adopting the entropy profile given by Eq. (5). Right panel: The solid line is our fit $\left(\chi^{2}=1.97 / 4\right)$ on adopting the entropy profile given by Eqs. (6) and (7). Note that the first bin covers the range out to about $0.04 h_{50}^{-1} \mathrm{Mpc}$ in Fig. 11.
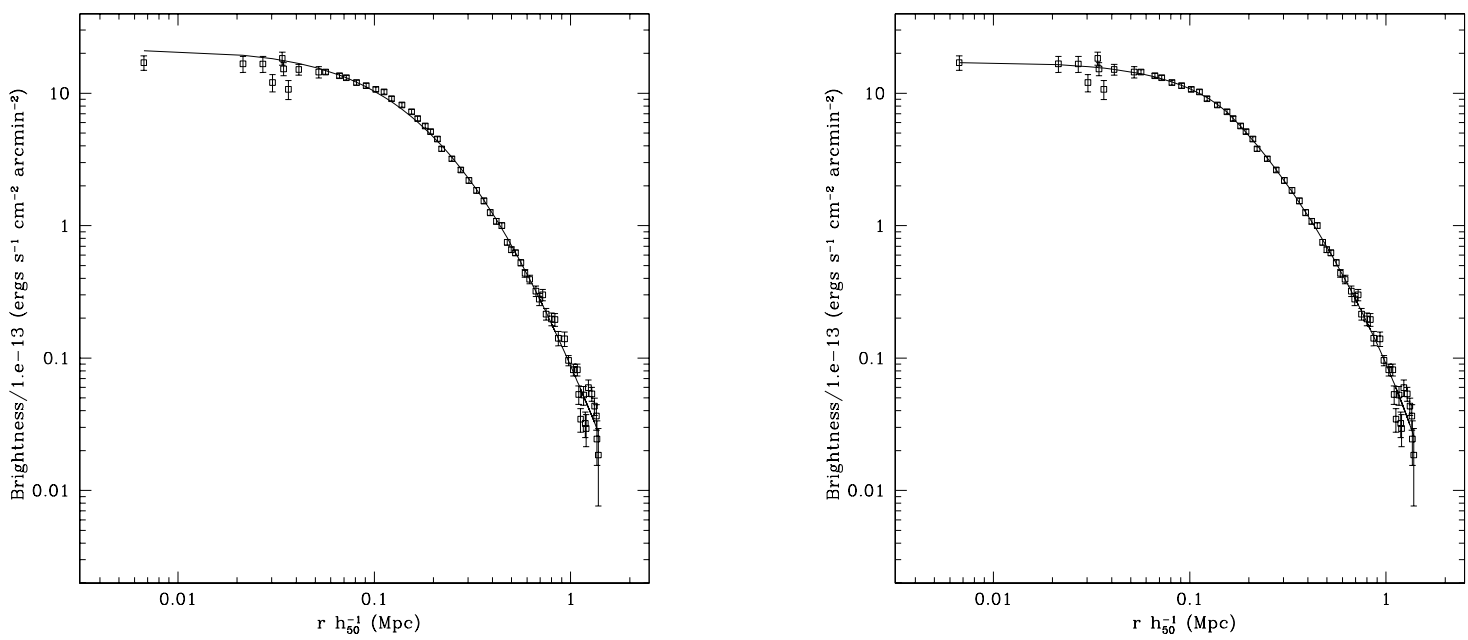

FIG. 11.- A644. Left panel: The solid line is our fit $\left(\chi^{2}=120.7 / 56\right)$ to the brightness profile measured by Mohr et al. (1999), on adopting the entropy profile given by Eq. (5). Right panel: The solid line is our fit $\left(\chi^{2}=76.3 / 55\right)$ on adopting the entropy profile given by Eq. (6) and (7).

central entropy level $k_{c}$ set by thermodynamical events, i.e., energy discharged and blasts driven by major mergers or AGN outbursts; in a number of cases the latter imprint substructure on the small scale $r_{f}$ comparable to $r_{m}$. A novel feature of the SM (relative to handy isothermal or polytropic $\beta$-models where the entropy is assumed to be a functional $k \propto n^{\Gamma-5 / 3}$ of the density, see Cavaliere \& Fusco-Femiano 1976) is constituted by the articulated radial entropy run entering Eq. (2).

We have collected in Table 1 the SM fitting parameters for all clusters in our set. By inspection it is apparent a correlation between the inner ICP profile type (marked by the CC/NCC tags) with the central entropy level $k_{c}$, the outer entropy slope $a$, and the DM concentration $c$; high values of $c$ and low values of $a$ and $k_{c}$ correspond to the CC class, while the opposite trend holds for NCC. We understand these trends in the framework of two-stage cluster formation (see $\S 1$ ) as follows. For

Eq. (2), except for the constant term within square brackets that is anyway negligible in cluster bodies (see CLFF09), and particularly so for the CCs. example, low values of $a$ correspond to high values of $b_{R}$ (see Eq. 4) owing to low values of $\Delta \phi$ (see Eq. 3); these are related to high concentrations $c=3.5\left(1+z_{t}\right)$ (see $\S 1$ and 2), which imply early transition redshifts $z_{t}$, i.e., an old age. We stress that in the six clusters considered here the outer parameters $a$ and $c$ turn out to be related to the inner parameter $k_{c}$ (and $r_{f}$ when applicable) in terms of the cluster age, as explained below.

Thus the CC clusters with their low value of $a$ appear to be generally older structures, currently in their stage of slow and smooth accretion, with the main action taking place in the outskirts under the form of calm entropy deposition by gravitational accretion shocks. Toward the center, the $\mathrm{CC}$ hallmark is constituted by a temperature peak overlapping (cf. Fig. 3 in CLFF09) the peak in $\sigma^{2}$ at $r_{m} \approx 10^{-1} R$ (cf. the profiles of A2199 and A2597 in Figs. 1-3). The condition for the peak to occur after the SM is a low value of the central entropy $\bar{k}_{c} \lesssim 3 \times 10^{-2}$, which comes to $30-50 \mathrm{keV} \mathrm{cm}{ }^{2}$. This behav- 
TABLE 1

FITTING PARAMETERS FROM THE SM ANALYSIS

\begin{tabular}{llcccccc}
\hline \hline Cluster & \multicolumn{1}{c}{ Class } & $c$ & $a$ & $\bar{k}_{c}\left[10^{-2}\right]$ & $r_{f}[\mathrm{kpc}]$ & $R[\mathrm{Mpc}]$ & $k_{B} T_{R}[\mathrm{keV}]$ \\
\hline A2199 & CC & $6.7_{-1.0}^{+1.0}$ & $0.95_{-0.01}^{+0.01}$ & $0.39_{-0.16}^{+0.16}$ & $\lesssim 2$ & $2.1_{-0.4}$ & $1.93_{-0.05}^{+0.05}$ \\
A2597 & CC & $7.2_{-5.2}^{+5.0}$ & $0.71_{-0.05}^{+0.05}$ & $0.21_{-0.12}^{+0.48}$ & $50_{-7}^{+7}$ & $1.9_{-0.4}^{+0.4}$ & $2.1_{-0.6}^{+0.8}$ \\
A1689 & CC & $13.6_{-4.3}^{+4.3}$ & $0.80_{-0.06}^{+0.06}$ & $2.4_{-0.8}^{+0.8}$ & $\lesssim 4$ & 2.1 & $4.4_{-0.6}^{+0.6}$ \\
A1656 & NCC & $3.0_{-0.8}^{+0.8}$ & $1.30_{-0.24}^{+0.50}$ & $10_{-1}^{+1}$ & $250_{-74}^{+44}$ & 2.2 & $5.7_{-1.0}^{+1.0}$ \\
A2256 & NCC (RCC) & $2.7^{+1.7}$ & $1.48_{-0.29}^{+0.35}$ & $6.2_{-3.1}^{+3.9}$ & $264_{-80}^{+102}$ & $2.2_{-0.3}^{+0.3}$ & $4.4_{-0.9}^{+0.9}$ \\
A644 & NCC (RCC) & $3.9_{-0.2}$ & $1.06_{-0.11}^{+0.11}$ & $0.7_{-0.1}^{+0.1}$ & $66_{-9}^{+8}$ & $2.1_{-0.4}^{+0.4}$ & $4.9_{-1.8}^{+1.8}$
\end{tabular}

Note. - The classification is taken from Molendi \& Pizzolato (2001), see also Henning et al. (2009). For A1689 and A1656, the value of the virial radius is taken from the literature (see text). The values of $k_{B} T_{R}$ are computed from Eq. (9), and are to be compared with those from the strong shock condition (see $\S 2$ and 3). For A644 the values of $\bar{k}_{c}$ and $r_{f}$ refer to the X-ray peak region; this explains the low $k_{c}$ value (see Fig. 10)

ior is highlighted in terms of $T(r) \propto k(r) n(r)^{2 / 3}$; as the ICP density $n(r)$ rises monotonically inward, $T(r)$ will peak and then decline toward the center as $k(r)$ decreases sharply toward a low central value $k_{c}$.

Such an inward decline of $T(r)$ to a low but finite central value $T_{c} \propto k_{c}^{0.35}$ links to a high density $n_{c} \propto k_{c}^{-1}$ to constitute the cool core, a feature of the non-radiative SM equilibrium (also present in simulations discussed by Borgani et al. 2008). As expanded upon by CLFF09, the SM does not include enhanced cooling, even less any related inflow; it rather focuses the conditions for enhanced radiation and fast cooling to set in on the timescale $t_{c} \approx 0.3\left(k_{c} / 15 \mathrm{keV} \mathrm{cm}^{2}\right)^{1.2} \mathrm{Gyr}$. This would lead to a cooling catastrophe (e.g., White \& Rees 1978; Blanchard et al. 1992), that may be stabilized by ICP condensing around and into a central massive galaxy to trigger accretion on the nuclear black hole. These conditions kindle up AGN activities that drive rising bubbles or even outgoing blastwaves, feed back entropy, and distribute it widely into the ICP (see Binney \& Tabor 1995; Ciotti \& Ostriker 2001; Cavaliere et al. 2002; Churazov et al. 2005; Lapi et al. 2005; Voit \& Donahue 2005; Tucker et al. 2007).

We have analyzed in detail the two CC clusters A2199 and A2597 with their inward decrease of the temperature. We have found low central entropy levels $k_{c} \lesssim 15 \mathrm{keV} \mathrm{cm}^{2}$ typical of CCs, with little or no need for an extended entropy floor. We have derived outer powerlaw slopes $a \lesssim 1$ (see Table 1 ), lower than the standard value 1.1 corresponding to the standard concentration $c \approx 4$.

This trend culminates with A1689, a cluster with a CC-like inner profile but featuring interesting outer peculiarities. On an empirical stand, our SM analysis confirms the results by Lemze et al. (2008) concerning the high halo concentration $c \approx 10$ (concurring with the gravitational lensing analysis by Broadhurst et al. 2008; Lapi \& Cavaliere 2009b) and involving the flat slope $a \approx 0.8$ for the outer entropy profile. But we go beyond, and show in terms Eqs. (3) and (4) why these values deviate from the standard ones $c \approx 4$ and $a \approx 1.1$, as spelled out above. In the same vein, CLFF09 find that a steeper density slope $g=3\left(a+b_{R}\right) / 5 \approx 2.4$ is to apply in the outskirts. The high concentration of A1689 implies this to be an old structure with the bulk region dating back to a transition epoch as early as $z_{t} \approx 1.5$. In fact, the feature common to CC clusters like A2199, A2597, and A1689 is constituted by low values of $a \lesssim 1$ and high values $c>4$ (see Table 1 ), that follow from their being generally old structures with shallow outer potential wells.

At the other extreme, the NCC clusters appear to be dy- namically young structures from our determination of DM concentration and slope in the outer entropy profile. For example, in A1656 (Coma Cluster) our SM fit requires a high value $a \approx 1.3$, and relatedly (see Eqs. 3 and 4) a low concentration $c \approx 4$ and young age $z_{t} \lesssim 0.5$. Toward the center, the NCC clusters are marked by a rising or flat temperature profile and by a generally flat brightness distribution. This occurs for central levels of $k_{c}$ exceeding some $50 \mathrm{keV} \mathrm{cm}^{2}$, and also points toward a thermodynamically young age for the ICP. In fact, frequent and intense merger/AGN activity is expected in these clusters observed in the aftermath of their fast initial collapse, with considerable residual occurrence of mergers and AGN outbursts that lead to large central injections of energy and entropy.

Such features are exhibited, in a sequence of increasing complexity, by A1656, A2256 and A644. Here, the SM elicits not only a high level, but also a pattern for the entropy deposited in the form of a floor extended out to $r_{f}$. This we interpret in terms of the stallation radius attained by a powerful, outbound blast either triggered by a major head-on merger (cf. simulations by Schindler et al. 2002, Vazza et al. 2009) or driven by a violent AGN outburst (see Forman et al. 2005; Cavaliere \& Lapi 2006; McNamara \& Nulsen 2007; Puchwein et al. 2008), before being degraded into adiabatic sound waves of the kind caught in action by Fabian et al. (2006) in the Perseus cluster. To reach $r_{f} \approx 250 \mathrm{kpc}$ it takes a rather extreme merger delivering about $10^{64} \mathrm{erg}$ and triggering a Sedov blastwave that expands as $R_{s} \propto E^{1 / 3} t^{2 / 3}$ with decreasing Mach number; alternatively, it takes an AGN outburst of about $10^{62}$ erg continuously driving a blastwave to expand at constant Mach number with $R_{s} \propto E^{1 / 3} t$, see Lapi et al. (2005) and Cavaliere et al. (2006) for details. As discussed in $\$ 4.4$ and 4.5, in the NCC clusters A1656 and A2256 analyzed here such values of $r_{f}$ are accompanied by evidence of ongoing mergers, hallmarks of a recent cluster formation.

This interpretation relates $r_{f}$ to the dating of the merger responsible for the energy/entropy input; the good performance of the SM implies such a time to be intermediate between the blast transit time $r_{f} / \mathcal{M} v_{s} \approx 10^{-1} \mathrm{Gyr}$ (see Cavaliere \& Lapi 2006), and the time $0.3\left(k_{c} / 15 \mathrm{keV} \mathrm{cm}^{2}\right)^{1.2} \approx 1$ Gyr needed by radiative cooling to erode an entropy floor of about $50 \mathrm{keV}$ $\mathrm{cm}^{2}$.

Such a timing also guarantees that an accurate description of the ICP thermodynamic state for both CC and NCC clusters is provided by the SM based on the hydrostatic equilibrium expressed by Eq. (1). To complete the issue, note that 
TABLE 2

FIT PARAMETERS OF EQS. (A1) AND

(A2)

\begin{tabular}{cccc}
\hline \hline$\alpha$ & $s$ & $u$ & $q$ \\
\hline 1.25 & 0.750 & 0.389 & 21.465 \\
1.26 & 0.756 & 0.399 & 14.374 \\
1.27 & 0.762 & 0.411 & 10.614 \\
1.28 & 0.768 & 0.423 & 8.316 \\
1.29 & 0.774 & 0.436 & 6.751 \\
& & & \\
\hline
\end{tabular}

not only the equilibrium of the ICP is somewhat faster to attain than the DM's (see Ricker \& Sarazin 2001; Lapi et al. 2005), but also that circularized data (integrated over annuli, see Snowden et al. 2008) tend to effectively smooth out local, limited deviations from spherical hydrostatics and to better agree with equilibrium.

How does the SM face the challenge of complexity posed by substructures as observed in A2256 and A644? Interestingly, we still obtain good fits if we extrapolate the SM out to, or perhaps beyond its literal limits, toward conditions where $T(r)$ varies on the scale $r_{f}$, or differs around two locations (cf. Figs. 8-11); these conditions highlight the capabilities of the SM as a mere fitting tool. By the same token, the SM provides sharp snapshots of physical conditions even when these are spatially complex (as for A644), and strongly suggests that they may be traced back to two merger outcomes: a 'hot spot' imprinted in A2256 to partially erase a previous cool state and to yield a nascent NCC; or a 'cold drop' imported into the hot medium of A644 that will offset cooling. Focusing on the cold component, such cases may be termed as RCCs for Remnant of Cool Cores.

In closing, we revert to Table 1 that provides an overall view of the SM parameters for the present cluster set, to stress the following points. First, columns 2, 3 and 4 visualize the sharp correlations among cluster classes (CC/NCC), basic properties of the DM halo (high/low concentration implying old/young dynamical age) and thermodynamic state of the ICP (low/high central entropy level, narrow/wide entropy floor, and steep/flat entropy slope). Second, columns 4 and 5 visualize how from the density and temperature distributions over the full data range we obtain entropy profiles of comparable quality to Zhang et al. (2008) and Cavagnolo et al. (2009). In particular, compared to the latter authors we find similar values of $k_{c}$ and somewhat lower values of $a$ (with the upper bound on $a$ discussed in $\S 2$ ); we note that our values are derived by directly fitting the primary X-ray observables
$S_{X}(w)$ and $T(w)$ with the SM, rather than deprojected profiles. Concerning virial radii, our values in column 7 systematically agree with literature evaluations; the agreements of the related virial masses are commented upon in $\S 4.3,4.4,4.5$. Finally, column 8 visualizes the boundary values $k_{B} T_{R}$ useful to introduce a discussion of the actual shock strengths related to the development of the outer halo and to preheating levels of the (Warm-Hot) IGM (see § 3).

To sum up, our analysis of several NCC and CC galaxy clusters has shown how effective is the tool constituted by our Supermodel. On using a simple formalism and a fast algorithm, this leads to accurately fit in terms of a few physical parameters the many data points concerning diverse temperature and brightness shapes to constitute the library indexed by Table 1 . Whence we extract sharp information concerning the evolutionary stage and the thermal history of clusters; in the inner regions these include the level $\left(k_{c}\right)$, pattern $\left(r_{f}\right)$ and timing of the entropy injections into the ICP, and in the outskirts the slope $(a)$ of the entropy deposited by accretion, simply related to the halo concentration $(c)$ and age $\left(z_{t}\right)$.

Finally, the Supermodel offers predictions as for the density and temperature profiles in the cluster outskirts out to the boundary $r \approx R$ facing the IGM; for example, within a surrounding supercluster enhanced IGM preheating may be expected to weaken the accretion shocks and lower the tail of the brightness and temperature profiles. Such outer regions challenge most current instruments but are coming of age with SUZAKU (see Bautz et al. 2009; George et al. 2009; Reiprich 2009), and will constitute a main target for the next generation X-ray telescopes planned to study low surface brightness plasmas, such as WFXT (see Giacconi et al. 2009; also http: / /wfxt.pha . jhu.edu/). These will open up the way to use galaxy clusters as probes of the surrounding Warm-Hot IGM, an interesting perspective that we will pursue in a following paper.

NOTE ADDED IN PROOF: The evidence concerning remnants of cool cores in NCC clusters is also discussed from an observational point of view by M. Rossetti \& S. Molendi (2009, A\&A, submitted).

Work supported by ASI and INAF. We acknowledge stimulating discussions with R. Giacconi, and informative exchanges with D. Buote and F. Gastaldello. We thank our referee for insightful comments, helpful toward improving our presentation. A.L. thanks SISSA and INAF/OATS for hospitality.

\section{APPENDIX}

\section{ANALYTIC FITS TO THE $\alpha$-PROFILES}

Here we provide analytic fits to the radial runs of both the density and the circular velocity in the DM halos following the (isotropic) $\alpha$-profiles.

It is convenient to express these in terms of the popular and flexible parametric expression for generic density profiles

$$
\bar{\rho}(\bar{r})=\frac{1}{\bar{r}^{s}}\left[\frac{1+w c^{u}}{1+w(c \bar{r})^{u}}\right]^{q}
$$

introduced by Zhao (1996) and constituting an extension of the empirical NFW (Navarro et al. 1997) formula (recall that $\bar{r}=r / R$ is the adimensional radial coordinate, while $c \equiv R / r_{-2}$ is the concentration parameter).

We cure the latter's unphysical features: diverging total mass and steep central cusp corresponding to angled potential, by deriving the parameters in Eq. (A1) directly from the Jeans equation and its derivatives, as shown by Lapi \& Cavaliere (2009a, see their Appendix A). In fact, by differentiating Eq. (A1) it is easy to see that the latter approach implies $w=-(2-s) /(2-s-q u)$. 
In addition, we find

$$
s=\gamma_{a} \quad, \quad u=\frac{6}{5} \frac{\kappa_{\text {crit }}}{\gamma_{0}-\gamma_{a}}-\frac{2}{3}\left(\gamma_{b}-\gamma_{a}\right) \quad, \quad q=\frac{\left(\gamma_{0}-\gamma_{a}\right)^{2}}{3 \kappa_{\text {crit }} / 5-2\left(\gamma_{0}-\gamma_{a}\right)^{2} / 3}
$$

here $\gamma_{a}=3 \alpha / 5, \gamma_{0}=6-3 \alpha, \gamma_{b}=3(1+\alpha) / 2$, and $\kappa_{\text {crit }}(\alpha) \simeq 8.23-4.44 \alpha$. In Table 2 we report the parameter determinations for several values of $\alpha$.

Using Eq. (A1), the function $\bar{v}_{c}^{2}(\bar{r})$ to be inserted in Eq. (2) of the main text may be explicitly expressed as

$$
{\overline{v_{c}}}^{2}(\bar{r})=\bar{r}^{2-s} \frac{{ }_{2} F_{1}\left[(3-s) / u, q, 1+(3-s) / u ;-w(c \bar{r})^{u}\right]}{{ }_{2} F_{1}\left[(3-s) / u, q, 1+(3-s) / u ;-w c^{u}\right]}
$$

in terms of the hypergeometric function ${ }_{2} F_{1}$; for a plot, see Fig. 1 in CLFF09.

The resulting fits to the density and circular velocity profiles of the $\alpha$-profiles hold to better than $15 \%$ in the whole range $10^{-2} \lesssim c \bar{r} \lesssim 10$. In particular, for $\alpha=35 / 27=1 . \overline{296}$ we recover the exact solution of the Jeans equation found by Dehnen $\&$ McLaughlin (2005).

\section{REFERENCES}

Adami, C., Biviano, A., Durret, F. \& Mazure, A. 2005, A\&A, 443, 17

Andersson, K.E., \& Madejski, G.M. 2004, ApJ, 607, 190

Balogh, M., McCarthy, I.G., Bower, R., \& Voit, G.M. 2007, in Heating versus Cooling in Galaxies and Clusters of Galaxies, ESO Astrophysics Symposia (Berlin: Springer-Verlag), p. 268

Bauer, F., \& Sarazin, C.L. 2000, ApJ, 530, 222

Bautz, M.W., et al. 2009, PASJ, in press [preprint arXiv:0906.3515

Berrington, R.C., Lugger, P.M., \& Cohn, H.N. 2002, AJ, 123, 2261

Binney, J., \& Tabor, G. 1995, MNRAS, 276, 663

Blanchard, A., Valls-Gabaud, D., \& Mamon, G. A. 1992, A\&A, 264, 365

Borgani, S., Diaferio, A., Dolag, K., \& Schindler, S. 2008, Sp. Sc. Rev., 134 269

Bourdin, H., \& Mazzotta, P. 2008, A\&A, 479, 307

Bridle, A.H., \& Fomalont, E.B. 1976, A\&A, 52, 107

Briel, U.G., et al. 2001, A\&A, 365, L60

Briel, U.G., et al. 1991, A\&A, 246, L10

Broadhurst, T., et al. 2008, ApJ, 685, L9

Buote, David A., Humphrey, P.J., \& Stocke, J.T. 2005, ApJ, 630, 750

Castander, F.J., et al. 2001, AJ, 121, 2331

Cavagnolo, K.W., Donahue, M., Voit, G.M., \& Sun, M. 2009, ApJS, 182, 12

Cavaliere, A., Lapi, A., \& Fusco-Femiano, R. 2009, ApJ, 698, 580 [CLFF09]

Cavaliere, A., \& Lapi, A. 2006, ApJ, 647, L5

Cavaliere, A., Lapi, A., \& Menci, N. 2002, ApJ, 581, L1

Cavaliere, A., \& Fusco-Femiano 1976, A\&A, 49, 137

Churazov, E., et al. 2005, MNRAS, 363, L91

Ciotti, L., \& Ostriker, J.P. 2001, ApJ, 551, 131

Clarke, T.E., \& Ensslin, T.A. 2006, AJ, 131, 2900

Clarke, T.E., et al. 2005, AAS 207, 1770

Clowe D., et al., 2006, ApJ, 648, L109

David, L.P., et al. 1993, ApJ, 412, 479

De Grandi, S., Ettori, S., Longhetti, M., \& Molendi, S. 2004, A\&A, 419, 7

Dehnen, W., \& McLaughlin, D.E. 2005, MNRAS, 363, 1057

Diemand, J., Kuhlen, M., \& Madau, P. 2007, ApJ, 667, 859

Ebeling, H., et al. 1996, MNRAS, 281, 799

Ettori, S. 2000, MNRAS, 311, 313

Fabian, A.C., et al. 2006, MNRAS, 366, 417

Forman, W., et al. 2005, ApJ, 635, 894

Gastaldello, F., Buote, D.A., Brighenti, \& F., Mathews, W.G. 2008, ApJ, 673, L17

Gavazzi, R. et al. 2009, A\&A, 498, L33

George, M.R., et al. 2009, MNRAS, 395, 657

Giacconi, R., et al. 2009, Science White Paper submitted to the US Astro2010 Decadal Survey [preprint arXiv:0902.4857]

Giacintucci, S., et al. 2008, ApJ, 682, 186

Giovannini, G., Feretti, L., \& Stanghellini, C. 1991, A\&A, 252, 528

Henning, J.W., Gantner, B., Burns, J.O., Hallman, E.J. 2009, ApJ, 697, 1597

Hoffman, Y., Romano-Diaz, E., Shlosman, I., \& Heller, C. 2007, ApJ, 671 1108

Johnstone, R.M., Allen, S.W., Fabian, A.C., \& Sanders, J.S. 2002, MNRAS, 336, 299

Kim, K.-T. 1999, JKAS, 32, 119.

Kubo, J.M. 2007, ApJ, 671, 1466

Lapi, A., \& Cavaliere, A. 2009a, ApJ, 692, 174
Lapi, A., \& Cavaliere, A. 2009b, ApJ, 695, L125

Lapi, A., Cavaliere, A., \& Menci, N. 2005, ApJ, 619, 60

Leccardi, A., \& Molendi, S. 2008, A\&A, 486, 359

Lemze, D., Barkana, R., Broadhurst, T.J., \& Rephaeli, Y. 2008, MNRAS, 386, 1092

Lokas, E.L., \& Mamon, G.A. 2003, MNRAS, 343, 401

Markevitch, M., \& Vikhlinin, A. 2007, Phys. Rep., 443,

Markevitch, M., et al. 2002, ApJ, 567, L27

McCarthy, I.G., et al. 2007, MNRAS, 376, 497

McCarthy, I.G., et al. 2008, MNRAS, 386, 1309

McNamara, B.R., \& Nulsen, P.E.J. 2007, ARAA, 45, 117

McNamara, B.R. et al. 2001, ApJ, 562, L149

Miller, N.A., Owen, F.N., \& Hill, J.M. 2003, AJ, 125, 2393

Mohr, J.J., Mathiesen, B., \& Evrard, A.E. 1999, ApJ, 517, 627

Molendi, S., \& Pizzolato, F. 2001, ApJ, 560, 194

Morris, R.G., \& Fabian, A.C. 2005, MNRAS, 358, 585

Navarro, J. F., et al. 2009, MNRAS, submitted [preprint arXiv:0810.1522|

Navarro, J.F., Frenk, C.S., \& White, S.D.M. 1997, ApJ, 490, 493

O'Sullivan, E., et al. 2005, MNRAS, 357, 1134

Piffaretti, R., Jetzer, P., Kaastra, J., \& Tamura, T. 2005, A\&A, 433, 101

Pratt, G.W., Böhringer, H, \& Finoguenov, A. 2005, A\&A, 433, 785

Puchwein, E, Sijacki, D., \& Springel, V., ApJ, 687, L53

Reiprich, T.H., et al. 2009, A\&A, 501, 899

Ricker, P.M., \& Sarazin, C. L. 2001, ApJ, 561, 621

Sarazin, C.L., \& McNamara, B.R. 1997, ApJ, 480, 203

Sarazin, C.L. 1988, X-ray Emission from Clusters of Galaxies (Cambridge: Cambridge Univ. Press)

Scannapieco, E., \& Oh, S.P. 2004, ApJ, 608, 62

Schindler, S. 2002, in Highlights in Astronomy, Vol. 12, ed. H. Rickman (San Francisco: ASP), p. 531

Snowden, S.L., Mushotzky, R.F., Kuntz, K.D., \& Davis, D.S. 2008, A\&A, 478,615

Sun, M., Murray, S.S., Markevitch, M., \& Vikhlinin, A. 2002, ApJ, 565, 867

Taylor, J.E., \& Navarro, J.F. 2001, ApJ, 563, 483

Tormen, G., Moscardini, L., \& Yoshida, N. 2004, MNRAS, 350, 1397

Tozzi, P., \& Norman, C. 2001, ApJ, 546, 63

Tucker, W., Tananbaum, H., \& Fabian, A. 2007, Sci. Am., 296, 42

Valageas, P., \& Silk, J. 1999, A\&A, 350, 725

Vass, I. M., Valluri, M., Kravtsov, A. V., \& Kazantzidis, S. 2009, MNRAS 395,1225

Vazza, F., et al. 2009, A\&A, submitted [preprint arXiv:0905.3169]

Voit, G.M. 2005, Rev. of Mod. Phys., 77, 207.

Voit, G.M., \& Donahue, M. 2005, ApJ, 634, 955

Wang, J., \& White, S.D.M. 2008, MNRAS, 396, 709

White, S.D.M., \& Rees, M.J. 1978, MNRAS, 183, 341

Wu, K.K.S., Fabian, A.C., \& Nulsen, P.E.J. 2000, MNRAS, 318, 889

Xue, Y.-J., \& Wu, X.-P. 2000, MNRAS, 318, 715

Xue, S.-J., \& Wu, X.-P. 2002, ApJ, 576, 152

Zhang, Y.-Y., et al. 2008, A\&A, 482, 451

Zhao, D.H., Mo, H.J., Jing, Y.P., \& Börner, G. 2003, MNRAS, 339, 12

Zhao, H. 1996, MNRAS, 278, 488 